\title{
PERIODIC SOLUTIONS OF PERTURBED CONSERVATIVE SYSTEMS
}

\author{
JAMES R. WARD ${ }^{1}$
}

\begin{abstract}
The existence of $2 \pi$-periodic solutions to the system $x^{\prime \prime}+$ $\operatorname{grad} G(x)=p(t, x), p$ being $2 \pi$-periodic in $t$, is established under conditions at infinity on the Hessian matrix of $G$. The condition used is weaker than earlier known conditions of a similar nature.
\end{abstract}

Introduction and preliminaries. This note concerns the differential equation

$$
x^{\prime \prime}+\operatorname{grad} G(x)=p(t, x),
$$

where $G \in C^{2}\left(R^{n}, R\right)$ and $p \in C\left(R \times R^{n}, R^{n}\right)$ is $2 \pi$-periodic in $t$ for each fixed $x \in R^{n}$. We give conditions "at infinity" on the Hessian matrix of $G(x)$ which imply the existence of at least one $2 \pi$-periodic solution of (I) when $p(t, x)$ is uniformly bounded.

The existence of $2 \pi$-periodic solutions to the equation

$$
x^{\prime \prime}+\operatorname{grad} G(x)=f(t)
$$

under condition (1.1) below has been the object of several interesting papers. Loud [8] initiated these studies with an investigation of a scalar version of (II). Leach in [7] extended these results. In [6] Lazer and Sánchez, using the Cesari alternative method, proved the following theorem.

Theorem A [LAZer-Sánchez]. Let $f \in C\left(R, R^{n}\right)$ be $2 \pi$-periodic. If $G \in$ $C^{2}\left(R^{n}, R\right)$ and there exist an integer $\tilde{n}$ and numbers $p$ and $q$ such that $\tilde{n}^{2}<p \leqslant q<(\tilde{n}+1)^{2}$ and if

$$
p I \leqslant\left(\partial^{2} G(a)\right) /\left(\partial x_{i} \partial x_{j}\right) \leqslant q I
$$

for all $a \in R^{n}$, then there exists a $2 \pi$-periodic solution of (II).

In other papers Lazer [5], Ahmad [1], and Kannan [3], and others, have extended these studies to show both existence and uniqueness for wider classes of systems. Recently Mawhin [9] has given a proof of Theorem A (also showing uniqueness) based on an abstract result of his which is in turn based upon a simple and elegant application of the contraction mapping principle. All of these papers have used condition (1.1) or a more general version of (1.1). Here we weaken (1.1) to hold "at infinity" and at the same time allow

Presented to the Society, January 4, 1978; received by the editors November 7, 1977. AMS (MOS) subject classifications (1970). Primary 34C25, 47H15; Secondary 34B15.

Key words and phrases. Periodic solutions, boundary value problems, nonlinear system of ordinary differential equations, Hilbert space methods.

'The author received support from a Pan American University Faculty Research Grant while writing this paper. 
our perturbation to depend upon $x$. Our methods are most closely related to those of Mawhin.

In this note $R$ denotes the real numbers, $|x|$ denotes the Euclidean norm of $x \in R^{n}, n \geqslant 1$ and $|A|$ will be used for the norm of a matrix $A$. Also $I$ denotes either the $n \times n$ identity matrix or the identity map on a Hilbert space. If $A$ and $B$ are two real $n \times n$ matrices by $A \leqslant B$ we mean $B-A$ is nonnegative definite.

\section{Statement and proof of the theorem.}

TheORem 1. Let $G \in C^{2}\left(R^{n}, R\right)$ and $p \in C\left(R \times R^{n}, R^{n}\right)$ with $p(t+$ $2 \pi, x)=p(t, x)$ for all $(t, x)$ and $p(t, x)$ uniformly bounded. Suppose there exist an integer $\tilde{n}$ and positive numbers $p, q$, and $r$ with $\tilde{n}^{2}<p \leqslant q<(\tilde{n}+1)^{2}$ such that whenever $a \in R^{n}$ and $|a| \geqslant r$

$$
p I \leqslant\left(\partial^{2} G(a) / \partial x_{i} \partial x_{j}\right) \leqslant q I .
$$

Then there is at least one $2 \pi$-periodic solution of (I).

Proof. Without loss of generality we may assume $\operatorname{grad} G(0)=0$ since otherwise we could subtract grad $G(0)$ from each side of the equation (I).

Let $S=L^{2}\left((0,2 \pi), R^{n}\right)$ be the Hilbert space of square integrable $R^{n}$ valued functions with the usual inner product

$$
(u, v)=\int_{0}^{2 \pi}\langle u(t), v(t)\rangle d t
$$

and norm denoted by $\|\cdot\|$. Here $\langle\cdot, \cdot\rangle$ denotes the usual inner product on $R^{n}$.

Let

$$
D=\left\{u \in S: u, u^{\prime} \in \mathrm{AC}, u^{\prime \prime} \in L^{2}, u(0)=u(2 \pi) \text { and } u^{\prime}(0)=u^{\prime}(2 \pi)\right\}
$$

and define $L: D \rightarrow S$ by $L u=u^{\prime \prime}$. Here AC means absolutely continuous.

It follows from (III), the fact that the Hessian $H(x)$ is symmetric, and the continuity of the Hessian $H(x)=\left(\partial^{2} G(x) / \partial x_{i} x_{j}\right)$ on $|x| \leqslant r$ that grad $G$ is globally Lipschitzian and there is a number $a>0$ such that $|\operatorname{grad} G(x)| \leqslant$ $a|x|$ for all $x \in R^{n}$. Therefore, if we define an operator $N$ on $S$ by $N(u)(t)=$ $\operatorname{grad} G(u(t))$ for $u \in S$ and $t \in[0,2 \pi]$ the operator $N$ will map $S$ continuously into itself. We define $M: S \rightarrow S$ by $M u(t)=p(t, u(t))$ for $u \in S$ and $0 \leqslant t \leqslant 2 \pi$. It is shown in [4, p. 22] that the continuity and boundedness of $P$ implies that $M$ is continuous and maps $S$ into itself. Any $2 \pi$-periodic solution of (I) is then a solution of the equation

$$
L x+N x=M x
$$

in $S$, and conversely.

Let $c=(p+q) / 2$; then $-c \in \rho(L)$ (the spectrum of $L$ is $\left\{-n^{2}: n \in Z\right\}$ ) and $(L+c I)^{-1}$ exists as a bounded linear operator on $S$ and it follows from known results concerning Green's functions (generalized to the case of 
uncoupled equations in $\left.R^{n}\right)$ that $(L+c I)^{-1}$ is compact [2, p. 192]. Equation (2.1) is equivalent to

$$
x=(L+c I)^{-1}[M+c I-N] x .
$$

We will use the Schauder fixed point theorem [11, p. 25] to show that (2.2) has a solution. The operator $M+c I-N$ is continuous and maps bounded sets in $S$ into bounded sets. The compactness of $(L+c I)^{-1}$ now implies that the operator $T$ defined by $T=(L+c I)^{-1}(M+c I-N)$ is completely continuous. If we can show that $T$ maps some closed ball in $S$ into itself we are done.

We first compute $\left\|(L+c I)^{-1}\right\|$. Because $L$ is selfadjoint and the interval $\left(-(\tilde{n}+1)^{2},-\tilde{n}^{2}\right)$ contains no numbers in the spectrum of $L$ we have as in [9]:

$$
\begin{aligned}
\left\|(L+c I)^{-1}\right\| & =\max \left\{\left(c-\tilde{n}^{2}\right)^{-1},\left((\tilde{n}+1)^{2}-c\right)^{-1}\right\} \\
& =[\min \{\ldots\}]^{-1} .
\end{aligned}
$$

Now let $u \in S$. We estimate $\|(M+c I-N) u\|$. Let $H(a)=$ $\left(\partial^{2} G(a) / \partial x_{i} \partial x_{j}\right)$ and $m$ a number with $m \geqslant|p(t, x)|$ for all $t, x$. We have

$$
\begin{aligned}
\|(M+c I-N) u\| & \leqslant\|M u\|+\|(N-c I) u\| \\
& \leqslant \sqrt{2 \pi} m+\|(N-c I) u\| .
\end{aligned}
$$

Further:

$$
\begin{aligned}
\|N u-c u\|^{2} & =\int_{0}^{2 \pi}|\operatorname{grad} G(u(t))-c u(t)|^{2} d t \\
& =\int_{0}^{2 \pi}\left|\left[\int_{0}^{1}(H(\lambda u(t))-c I) d \lambda\right] u(t)\right|^{2} d t \\
& \leqslant \int_{0}^{2 \pi}\left[\int_{0}^{1}|H(\lambda u(t))-c I| d \lambda\right]^{2}|u(t)|^{2} d t
\end{aligned}
$$

by Taylor's theorem and since grad $G(0)=0$.

We make the observation that whenever $|\lambda u(t)| \geqslant r$ we have as in [9] by the symmetry of the matrix $\left(\partial^{2} G(\lambda u(t)) / \partial x_{i} \partial x_{j}\right)=H(\lambda u(t))$ :

$$
\begin{aligned}
|H(\lambda u(t))-c I| & =\sup _{|y|=1}\langle H(\lambda u(t)) y-c y, y\rangle \\
& \leqslant \max \{(q-c),(c-p)\}=\beta .
\end{aligned}
$$

Let $z(\lambda u(t))=|H(\lambda u(t))-c I|$ and choose $\varepsilon>0$. Let $E_{1}=\{(\lambda, t):|\lambda u(t)|$ $\leqslant r$ and $0 \leqslant \lambda \leqslant \varepsilon\}, E_{2}=\{(\lambda, t):|\lambda u(t)| \leqslant r$ and $\varepsilon \leqslant \lambda \leqslant 1\}$, and $E_{3}=$ $\{(\lambda, t):|\lambda u(t)|>r\}$. We have: 


$$
\begin{aligned}
\|N u-c u\|^{2} \leqslant & \int_{0}^{2 \pi}\left[\int_{0}^{1} z(\lambda u(t)) d \lambda\right]^{2}|u(t)|^{2} d t \\
= & \int\left[\int_{E_{1}} z(\lambda u(t)) d \lambda\right]^{2}|u(t)|^{2} d t \\
& +\int\left[\int_{E_{2}} z(\lambda u(t)) d \lambda\right]^{2}|u(t)|^{2} d t \\
& +\int\left[\int_{E_{3}} z(\lambda u(t)) d \lambda\right]^{2}|u(t)|^{2} d t \\
\leqslant & \varepsilon^{2} k^{2}\|u\|^{2}+k^{2} \int_{\{t:|u(t)|<r / \varepsilon\}}|u(t)|^{2} d t+\beta^{2}\|u\|^{2} \\
\leqslant & \varepsilon^{2} k^{2}\|u\|^{2}+k^{2} r^{2} / \varepsilon^{2}+\beta^{2}\|u\|^{2}
\end{aligned}
$$

where $k=\sup \{|H(x)-c I|:|x| \leqslant r\}$. If $\|u\| \geqslant r k / \varepsilon^{2}$ we have from (2.6):

$$
\begin{gathered}
\|N u-c u\|^{2} \leqslant \varepsilon^{2}\left(k^{2}+1\right)\|u\|^{2}+\beta^{2}\|u\|^{2}, \\
\|N u-c u\| \leqslant\left[\varepsilon^{2}\left(k^{2}+1\right)+\beta^{2}\right]^{1 / 2}\|u\| .
\end{gathered}
$$

Since $\beta=\max \{(q-c),(c-p)\}$ and

$$
\left\|(L+c I)^{-1}\right\|=[\min \{\ldots\}]^{-1}
$$

and $c=(p+q) / 2$ with $\tilde{n}^{2}<p \leqslant q<(\tilde{n}+1)^{2}$ it is clear that

$$
\beta\left\|(L+c I)^{-1}\right\|<1 \text {. }
$$

Thus we may choose $\varepsilon=\varepsilon_{1}$ so small that whenever $\|u\| \geqslant k r / \varepsilon_{1}^{2}$ we have by (2.7) and (2.8) that there is a number $d, 0<d<1$, such that

$$
\left\|(L+c I)^{-1}\right\| \cdot\|N u-c u\| \leqslant d\|u\| .
$$

We can now show that the operator $T=(L+c I)^{-1}(M+c I-N)$ maps a closed ball of the form $B_{n}=\{u \in S:\|u\| \leqslant n\}, n$ a positive integer, into itself. If not, then we can find a sequence $\left\{x_{n}\right\}$ in $S$ with $x_{n} \in B_{n}$ and $\left\|T x_{n}\right\|>n$. The sequence $\left\{x_{n}\right\}$ must tend to infinity in norm, since otherwise $T$ would be mapping a bounded set onto an unbounded one. Hence $\left\|x_{n}\right\| \rightarrow$ $\infty$ and $\left\|x_{n}\right\|>r k / \varepsilon_{1}$ for all but finitely many $n$. By (2.4) and (2.9) we have for large $n$ :

$$
\left\|x_{n}\right\| \leqslant n<\left\|T x_{n}\right\| \leqslant d\left\|x_{n}\right\|+\sqrt{2 \pi} m\left\|(L+c I)^{-1}\right\|
$$

and

$$
\left\|x_{n}\right\| \leqslant(1-d)^{-1} \sqrt{2 \pi} m\left\|(L+c I)^{-1}\right\| .
$$

This contradicts the unboundedness of the $\left\{x_{n}\right\}$. Hence $T$ maps some ball $B_{k}$ into itself. By the Schauder theorem there exists $x_{0} \in B_{k}$ with $x_{0}=T x_{0}$. Hence $x_{0} \in D(L)$ and 


$$
L x_{0}=-N x_{0}+M x_{0} .
$$

Since $x_{0}(t)$ is continuous on $[0,2 \pi]$ and

$$
x_{0}^{\prime \prime}(t)=-\operatorname{grad} G\left(x_{0}(t)\right)+p\left(t, x_{0}(t)\right)
$$

it follows that $x_{0}^{\prime \prime}(t)$ is continuous on $[0,2 \pi]$. The function $x_{0}(t)$ may now be extended periodically to all of $R$. This extension is clearly a periodic solution of (I) on all of $R$. This completes the proof of the theorem.

REMARK 1. The uniform boundedness of $p(t, x)$ was not essential, and it is clear that the theorem remains true if $p(t, x)$ is sublinear, i.e., if $\lim |p(t, x)| /|x|=0$, as $|x| \rightarrow \infty$, the convergence being uniform in $t$.

REMARK 2. This method is not restricted to the periodic problem, but could also be used to handle other problems.

REMARK 3. Reissig [10] has extended Mawhin's approach to the equation

$$
x^{\prime \prime}+C x^{\prime}+\operatorname{grad} G(x)=e(t)
$$

with $C$ symmetric. His results can be combined with the methods of this paper to give similar results to our Theorem 1 for the equation

$$
x^{\prime \prime}+C x^{\prime}+\operatorname{grad} G(x)=p(t, x) \text {. }
$$

The author is indebted to the referee for this observation.

\section{REFERENCES}

1. S. Ahmad, An existence theorem for periodically perturbed conservative systems, Michigan Math. J. 20 (1973), 385-392.

2. E. A. Coddington and N. Levinson, Theory of ordinary differential equations, McGraw-Hill, New York, 1955.

3. R. Kannan, Periodically perturbed conservative systems, J. Differential Equations 16 (1974), 506-514.

4. M. A. Krasnosel'skii , Topological methods in the theory of nonlinear integral equations, Macmillan, New York, 1964.

5. A. C. Lazer, Application of a lemma on bilinear forms to a problem in nonlinear oscillations, Proc. Amer. Math. Soc. 33 (1972), 89-94.

6. A. C. Lazer and D. A. Sánchez, On periodically perturbed conservative systems, Michigan Math. J. 16 (1969), 193-200.

7. D. E. Leach, On Poincare's perturbation theorem and a theorem of W. S. Loud, J. Differential Equations 7 (1970), 34-53.

8. W. S. Loud, Periodic solutions of nonlinear differential equations of Duffing type, Proc. U.S.-Japan Seminar on Differential and Functional Equations (Minneapolis, Minn., 1967), Benjamin, New York, 1967, pp. 199-224.

9. J. Mawhin, Contractive mappings and periodically perturbed conservative systems, Scripta Fac. Sci. Natur. UJEP Brunensis-Math. 12 (1976), 67-74.

10. R. Reissig, Contraction mappings and periodically perturbed nonconservative systems, Atti Accad. Naz. Lincei Rend. Cl. Sci. Fis. Mat. Natur. (8) 58 (1975), 696-702.

11. D. A. Smart, Fixed point theorems, Cambridge Univ. Press, Cambridge, 1975.

Department of Mathematics, Pan American University, Edinburg, TeXas 78539 